\title{
Causes of Hospitalization Among Extra-European Union Children in a Large Hospital of Northern Italy, in a Five-Year Observation Period
}

\author{
Sergio Sabbatani ${ }^{1}$, Elena Baldi ${ }^{2}$ and Roberto Manfredi $^{1}$ \\ ${ }^{1}$ Department of Clinical and Experimental Medicine, Division of Infectious Diseases, “Alma Mater Studiorum” University of Bologna, S. \\ Orsola-Malpighi Hospital; ${ }^{2}$ Department of Hygiene and Public Health, "Alma Mater Studiorum” University of Bologna; Bologna, Italy
}

\begin{abstract}
The hospitalizations of 1,239 patients aged 14 years or less and immigrated from extra-European Union countries in Italy were assessed in the 6-year period, from 1999 to 2004. The main demographic and clinical features were analyzed according to several variables, also distiguishing patients aged less than one year, from those aged 1-14 years. The introduction of a deed of indemnity law in 2001 profoundly changed the pattern of admissions and health care needs and exploitation during subsequent years, leading to a massive regularization of clandestine immigrants. Key-Words: Children, diseases, extra-European Union, hospital admission, temporal trend.
\end{abstract}

Since mid-eighties, new and unexpected migration waves involved Italy, which during the subsequent years substantially acted on the entire social context, related to the extent of this immigration phenomenon, which brought to Italy an estimated number of three million people, with around 400,000 of them remaining for a long period in a clandestine status $[1,2]$.

\section{Materials and Methods}

The aim of our study is to assess all hospitalizations carried out at our tertiary care reference Hospital (S. Orsola-Malpighi Hospital, Bologna, Italy; a 1,800-bedded downtown facility), from the year 1999 (January 1) to 2004 (December 31), by extraEuropean Union (EU) children aged 14 years or less, in order to indirectly evaluate the prominent health problems of young immigrants and their children, by looking at admissions, discharge diagnoses, and their multiple correlates. From "foreign" citizens, patients coming from the S. Marino Republic, and those coming from extra-EU, but industrialized and technologically advanced countries were ruled out (e.g. USA, Canada, Israel, and Australia), in order to focus our attention on subjects coming or immigrating from developing areas of the world. Statistical data analysis was performed with computer programs belonging to the SPSS 12.0 software package, relying on usual indicators of frequency synthesis, and hypothesis testings like Pearson chi-square test or Fisher exact test, and chi-square per trend, with significant $\mathrm{p}$ values posed at $\mathrm{p}<.05$.

\section{Results}

Among 7,247 comprehensive hospitalizations of extra-EU patients examined through our informatized databases, in 9.4\% of cases the citizenship was not recorded, due to irregular or incomplete accompanying documentation. The admission of

Received on 27 September 2006; revised 18 December 2006.

Address for correspondence: Dr. Roberto Manfredi. Infectious Diseases, University of Bologna. S. Orsola Hospital, Via Massarenti 11, I-40138 Bologna, Italy. Telephone: +39-051-6363355. Telefax: +39-051343500. E-mail: roberto.manfredi@unibo.it.

The Brazilian Journal of Infectious Diseases 2007;11(1):6-8. (C) 2007 by The Brazilian Journal of Infectious Diseases and Contexto Publishing. All rights reserved. clandestine patients, which was a very common event during the years 1999-2000, had a drastic drop thereafter due to the relevant deed of indemnity Italian law (so-called Bossi-Fini law, 2001), which allowed to overcome this unfavorable situation. In fact, in the year 2002 compared with 1999 we observed a significant reduction of hospitalizations of clandestine children (chi-square 203.261; $\mathrm{p}<.001$ ). When analyzing separately hospitalizations regarding neonates and individuals aged less than one year, compared with patients aged 2-14 years, $43.7 \%$ of younger chidren had regularized parents ( $47 \%$ of these patients were females).

When excluding from the present evaluation adult patients, only the 1,239 hospitalizations regarding children aged 14 years or less were considered. Even 780 patients (62.9\%) were aged 12 months or less ( $46 \%$ of them were females), while the remaining 459 patients were aged from $>1$ to 14 years (the female gender accounted for $41 \%$ of these cases). In order to have a more detailed picture of health care needs of this special patient population, we considered overall diagnosis-related group (DRG) clinical diagnoses (which were often multiple for each single admission), instead of the main discharge diagnosis. In fact, each single discharge diagnosis produced one single DRG in $37 \%$ of cases, two DRGs in $16.5 \%$ of patients, three DRGs in $8.1 \%$, four DRGs in $4.1 \%$, and five or more DRGs in $2.5 \%$ of examined children. As a consequence, the information coming from the main discharge diagnosis of 1,239 hospitalizations led to 1,511 overall, and more detailed and significant DRGs (22\% more compared with simple discharge diagnoses).

Starting from the year 2002, a drop of overall childbirths occurred (chi-square 224.884; $\mathrm{p}<.001$ ), while in the three preceding years (1999-2001) a permanent trend towards an increased number of births was observed. While until 2001 the increased natality rate was attributable to immigrants coming from Asia and Northern Africa, after the year 2002 a slight increase occurred only for children of citizens coming from Eastern Europe. Children born to irregular immigrants virtually disappeared after the year 2002, and in our survey they represent $28.7 \%$ of the whole examined pediatric population.

In order to assess the trend of childbirth compared with that of aborptions, a chi-square test for trend of overall inpatients and the three predominant subgroups of immigrants 
(Asia, Eastern Europe, and Northern Africa), was performed. The result was statistically significant in overall examined population, with a chi-square of 119.129 , leading to a p value $<0.001$. The Picture 1 shows the progressive decrease of odds ratio (OR) since the year 2001, when the indemnity Italian law was introduced for clandestine immigrant citizens. A similar tendency is also retrieved among population coming from Eastern Europe (chi-square 25.604; p. $<02$ ), and those coming from Northern Africa (chi-square 17.54; $\mathrm{p}<.01$ ), while a less prominent trend is typical of Asian migrants (chi-square 7.7; $\mathrm{p}<.01$ ) (Figure 1).

Among aborptions, voluntary ones represent 436 cases (e.g. $47.9 \%$ of overall aborptions). While aborptions were an infrequent cause of admission in the year 1999 (25 cases) and 2000 (15 episodes), a ten-fold increase was observed in the year 2002 versus 2001 (148 versus 14, respectively). These procedures were prevailingly requested by women from Eastern Europe (97 cases of 148: 65.5\%), while the picture remained steadily over 100 episodes per year during the subsequent period: 109 episodes in the year 2003 (65.1\% involving Eastern European women), and 125 in the year 2004 (81.6\% requested by women from Eastern Europe) (Figure 2).

After excluding admissions directly or indirectly linked to childbirths, the diseases responsible for hospital admission of foreign (extra-EU) children aged one year or less were predominantly infectious-parasitic in origin (28.8\%), followed by genetical-congenital disorders (22\%), and dysmetabolic, functional, or organic illnesses involving different body organs and sites (20.9\%). The $5 \%$ of admissions due to nutritional deficiencies, deserves particular attention and concern. Among infectious disorders, an etiological agent was not determined in $12.9 \%$ of episodes, while an ascertained bacterial

Figure 1. Odds ratio (OR) per trend of childbirths versus voluntary aborptions, during the six examined years of hospitalization at our Hospital (1999 to 2004). Overall data are presented, together with those of the three main considered population subsets.

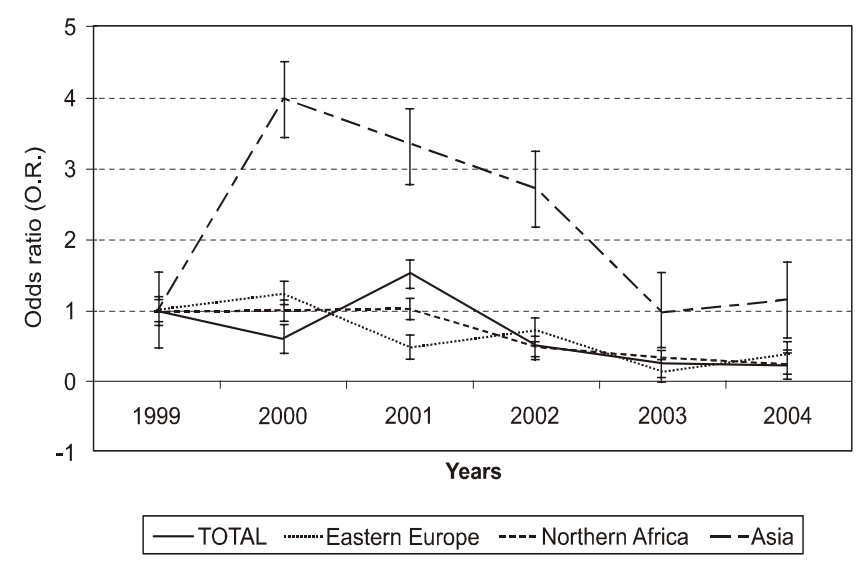

or viral origin was established in $4.6 \%$ and $2.6 \%$ of cases, respectively. In detail, two cases of HIV infection were found, while tuberculosis and syphilis occurred in one case each. Although no significant difference was found as for country of origin, children coming from Eastern Europe were burdened by an increased rate of infectious disorders leading to hospitalization (39 out of 110:35.4\%), as occurred in the past for clandestine children, too (37 episodes out of 110: 32.7\%). When assessing genetical-congenital disorders, again children coming from Eastern Europe (36\%), and those with nonregularly immigrated parents (34.9\%), prevailed. A frank malnutrition requiring hospital admission involved predominantly neonates and children from Eastern Europe (72.3\% of malnourished children), and clandestine ones (20.7\%).

Among hospitalized children aged more than one year, an increased number of admissions was observed since the year 2002 (chi-square 268.107; $p<.001$. Again, this increase was mainly attributable to Eastern Europeans children (44.1\%). In this older patient group (from $>1$ to 14 years of age), the broader spectrum of diseases responsible for hospitalization included dysmetabolic, functional, or organic disorders $(24.7 \%$ as a whole), followed by genetical-congenital diseases (15.7\%), and infectious and parasitic illnesses (13.5\% of episodes). Once again, internal medicine and dysmetabolic disease occurred more frequently in children coming from Eastern Europe (41.5\%), followed by children of clandestine families (24\%), and children coming from Northern African countries (14.1\%). Among infectious disorders, in $5.8 \%$ of cases the etiological agent could not be identified, while 10 cases of tuberculosis (7 coming from Eastern Europe), four patients with hepatitis B, two cases each of HIV infection and syphilis,

Figure 2. Odds ratio (OR) for trend of childbirths versus voluntary or spontaneous and voluntary aborptions, during the six examined years of hospitalization at our Hospital (1999 to 2004).

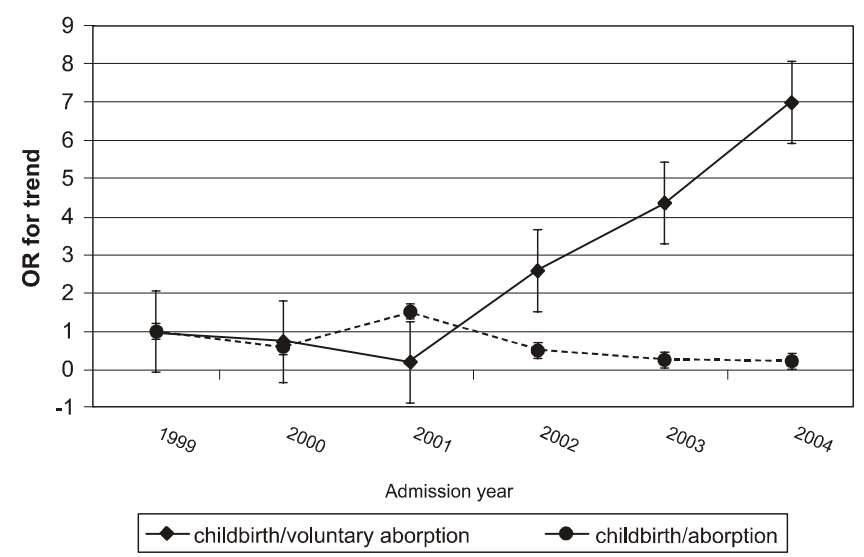


and one case of hepatitis C, were detected. Geneticalcongenital illnesses mainly involved Eastern European children (36\%), Northern Africans (23.2\%), and clandestines as a whole (34.9\%). Among children aged $1-14$ years versus younger ones, the malnourishement virtually disappeared as a cause of admission, when excluding two cases of patients immigrated from Eastern Europe. Finally, as a characteristic of the pediatric facilities available at our Hospital, even $14.4 \%$ of admissions were due to pediatric malignancies, and $9.1 \%$ of hospitalizations were directly linked to the delivery of hematological cytotoxic chemotherapy or radiotherapy.

\section{Discussion}

In conclusion, our preliminary assessment seems to show that a rapid modification of health care assistance of extraEuropean Union citizens immigrated from developing countries to Italy, occurred during the past six years (1999 to 2004), while profound differences are evident according to patient's age, gender, and related disorders, as well as the condition of regular or clandestine immigrants [3-6]. When assessing our experience carried out in Northern Italy, we can claim that either diseases features, or temporal trend of admission, or country of provenience of children, all these characteristics were profoundly affected by the 2001 modifications of Italian law, which allowed the regularization of hundred thousands of clandestine immigrants in our country. In particular, this legislative measure led to immediate and significant consequences on either childbirths, voluntary aborptions, and hospitalization rate and causes, by reducing drastically the amount of clandestine children, which were burdened by a broad spectrum of disadvantaged conditions. As a consequence of increased regularization, starting from 2002 a significant increase of voluntary aborption performed by public structures was reported among Eastern Europeans and North Africans. The occurrence of tuberculosis (a diffusive infectious disorders which also requires appropriate public health measures) was prevailingly attributable to children from Eastern Europe, while malnourishment was found in the same population cluster, while before 2001 it was a more common feature of clandestine immigrants as a whole, while during recent years this worrying phenomemon tended to a complete disappearance. The population cluster which was more heavily interested by dysmetabolic, functional, organic, genetical, and infectious disorders after the year 2002 is that coming from Eastern Europe (the last one coming to Italy after migration processes), while until the year 2001 these conditions were more common among children from irregular (clandestine) families. Appropriately targeted civil and health care actions are recommended especially when Eastern Europe families are involved, since this last population cluster seems now affected by the most prominent health care needs, often satisfied by the resort to Hospital institutions, especially through Emergency Rooms. As a consequence of the 2001 Italian indemnity law, the regularization obtained by formerly clandestine extra-EU immigrants, allowed the emergence of an "underground" and poorly known world of pediatric disease and aborption which were neither evident nor considered previously, since all these events occurred outside the control and action of the national health care services.

From an health care and social perspective, although a proportionally low rate of infectious illnesses occurred during time, the possibility to attribute them to patients of ascertained identity and housing, makes now possible to trace index cases, towards well-planned and effective therapeutic and preventive interventions [2-6]. The need of rely on a continued availability of adequate cultural mediators who could help understanding the health care needs of these disadvantaged patients is strongly needed, and a competence beyond the simple language aid is expected to grow. Furthermore, a first-line health care assistance network should be appropriately strengthened, by organizing primary care and multi-specialistic outpatient services easily accessible to immigrants, in order to offer immediate help, and avoid an inappropriate resort to Emergency Rooms and Hospitals admissions. In particular, the organization of specialized obstetric-gynecological consulting rooms should be urgently set up, to support female immigrates in caring their health, and operating a familyplanning counseling aimed at following women and their families, and avoiding undesired pregnancies and the frequent resort to medical-assisted aborption. Certainly the massive regularization of clandestine immigrates which occurred approximately four year ago (early 2002) allowed to ameliorate overall health conditions, by acting favorably also on the public health management. In fact, the modified regulatory laws prompted the emerging of epidemiological and health care problems, including those related to obstetricalgynecological instances (e.g. the phenomenon of voluntary pregnancy interruption in some clusters of immigrated women), and infectious disease, too, which remained sumerged until the year 2002.

\section{References}

1. Caritas Italiana, Rome. Migrantes. Statistical dossier immigration year 2003. XIII Report on Immigration. Available online at: www.caritasitaliana.it/immigrazione

2. Manfredi R., Calza L., Chiodo F. HIV disease among immigrants coming to Italy from outside of the European Union: a casecontrol study of epidemiological and clinical features. Epidemiol Infect 2001;127:527-33.

3. Sabbatani S, Cannella B, Zanna S. Dati clinico-epidemiologici su una casistica di pazienti extracomunitari di Bologna ricoverati per patologie infettive. Giornale Italiano di Medicina Tropicale 2000;5:57-65.

4. Stampi S., Matteini P., Zanetti F., Baldi E. Immigration and health: observational study concerning the foreign children attending the Bologna Community pediatric service. Ann Ig 2003;15:261-70.

5. Scotto G., Saracino A., Pempinello R., et al. SIMIT epidemiological multicentric study on hospitalized immigrants in Italy during 2002. J Immigr Health 2005;7:55-60.

6. Gabutti G., Fedele A., Aprile V., et al. Immigration flows and new epidemiological evidence in Southern Italy. Vaccine 2003;21:399-400. 